Concept ual desi gn of a compact hel i cal fusi on react or FFHR-1 f or the early demonst $r$ at $i$ on of year-I ong el ectric power gener at i on

\begin{tabular}{|l|l|}
\hline $\begin{array}{l}\text { j our nal or } \\
\text { publ i cat i on ti t l e }\end{array}$ & Nucl ear Fusi on \\
\hline vol une & 59 \\
\hline number & 7 \\
\hline page r ange & 076030 \\
\hline year & $2019-06-10$ \\
\hline NAl S & 11709 \\
\hline URL & ht t p: //hdl . handl e. net /10655/00012527 \\
\hline
\end{tabular}

doi: https://doi.org/10.1088/1741-4326/ab15c3 


\title{
Conceptual Design of a Compact Helical Fusion Reactor FFHR-c1 for the Early Demonstration of a Year-long Electric Power Generation
}

\author{
T. Goto, J. Miyazawa, H. Tamura, T. Tanaka, R. Sakamoto, C. Suzuki, R. Seki, S. Satake, \\ M. Nunami, M. Yokoyama, N. Yanagi, A. Sagara, The FFHR Design Group \\ National Institute for Fusion Science (NIFS), Toki, Gifu, 502-5292 Japan \\ E-mail: goto.takuya@nifs.ac.jp
}

\begin{abstract}
Conceptual design of a compact LHD-type helical fusion reactor FFHR-c1 has been conducted. This design focuses on a year-long electric power generation with as small a reactor size as possible by adopting the operation with auxiliary heating and innovative ideas for the design of superconducting magnet, divertor and blanket system. The primary design parameters, which have been selected by the extrapolation from the previous design FFHRd1B using simple scalings, has been examined by the systems code and the 1D integrated physics analysis code from the viewpoint of plant power balance and the achievable plasma operation regime. The design feasibility of the proposed design point has been confirmed with the physics parameters that are consistent with the LHD experiment. Further improvement of the plasma performance is expected by the optimisation of the helical coil winding law. Though intensive R\&Ds are needed to realise the innovative ideas of the engineering design, this design ensures the path to helical commercial power plants and provides more options and increase the probability of solving critical issues of fusion reactors.
\end{abstract}

Keywords: heliotron, reactor design, year-long operation, net electric power generation

\section{Introduction}

Helical systems inherently have a suitable feature as a future fusion power plant: flexible plasma operation regime that is free from the limitation caused by the plasma current, easiness in a steady-state operation and high plant efficiency because of no need of the plasma current drive. Among several configurations, heliotron system with two continuous helical coils has several advantages from the viewpoint of reactor design. For example, there are a large number of experimental data that has been accumulated by the operation of the Large Helical Device (LHD) [1], resulting in the core plasma physics design with a high certainty. Built-in and rigid divertor field structure enables a flexible divertor design. Relatively simple shape of the coil and the supporting structure and large ports enables a flexible approach of the construction and maintenance.

Based on these advantages, the conceptual design activity of the LHD-type helical reactor FFHR has been continued [2]. Although the primary design parameters (reactor size, magnetic field strength and fusion power output) have been re-examined several times during the long-term activity, the design has consistently maintained the following two policies: significant electric power production with a selfignited plasma and long plant lifetime over 30 years.

This activity has shown the design feasibility of the helical fusion reactor that can be operated with a 3 GW-class fusion output for a long period of $\sim 30$ years with the LHD-type configuration and the ITERrelevant technology. On the other hand, achievable plasma operation regime has been clarified by a detailed examination of the core plasma design. The achievable fusion gain is $\sim 10$ when the operation regime is restricted by the physics parameters that have already been confirmed by the LHD experiment even if a high-field option with magnetic field on the centre of helical coil winding, $B_{\mathrm{c}} \sim 6 \mathrm{~T}$, is adopted. In the meantime, several innovative ideas have been proposed, for example, "joint-winding" method of helical coils using the high temperature superconductor (HTS) to shorten the time for the winding [3], 
blanket space enlargement ( 15\%) by placing supplementary helical coils (NITA coils) to secure the tritium breeding and neutron shielding performance [4], adoption of liquid metal ergodic limiter/divertor system to enable high heat/particle load accommodation $\left(>100 \mathrm{MW} / \mathrm{m}^{2}\right)$ and easy maintenance [5], and proposal of cartridge-type blanket modules to achieve construction and maintenance without complicated works inside the vacuum vessel [6]. Though these innovative concepts have been proposed to overcome the engineering difficulties in the design of FFHR-d1, these concepts also enable a compact reactor design if focusing on the production of positive net electric power $\left(P_{\mathrm{e}, \text { net }}>0\right)$ by allowing operation with auxiliary heating and a shorter reactor lifetime due to the higher neutron flux to the superconducting coils. Therefore, a new design concept, FFHR-c1, which aims at a year-long steadystate operation with self-generated electricity and tritium fuel, has been proposed [7]. In this study, design window analysis for the confirmation of the proposed design point and integrated physics analysis of the core plasma for the examination of the achievable fusion gain were conducted. The method and result of the design window analysis are described in Section 2. The method and result of the integrated physics analysis are described in Section 3.

\section{Design window analysis}

The candidate design point of FFHR-c1 has been proposed with the major radius of the helical coil of $R_{\mathrm{c}}=10.92 \mathrm{~m}$ and the magnetic field strength at the winding centre of the helical coil of $B_{\mathrm{c}}=7.3 \mathrm{~T}$ according to the gyro-Bohm scaling of the core plasma performance and a simple scaling for the stored magnetic energy of the superconducting system $\left(R_{\mathrm{c}}{ }^{3} B_{\mathrm{c}}{ }^{2}\right)$ [7]. To examine the feasibility of this design point from the viewpoint of plant power balance, design window analysis was conducted using the systems code for LHD-type helical reactors, HELIOSCOPE [8]. Regarding the on-site power consumption of the plant equipment, plasma heating system (ECRH is assumed), cryogenic system and coolant pumping system of blanket and divertor modules are considered with following assumptions: neutron energy multiplication in the blanket module of 1.34 , power recovery factor at the divertor module of 0.25 , wall-plug efficiency of the ECRH system of 0.39 and efficiency of the cryogenic system of 0.015 . Figure 1(a) shows the calculation result in the case of the current density of the helical coils of $j_{\mathrm{c}}=25 \mathrm{~A} / \mathrm{mm}^{2}$ without NITA coils. This current density can be achieved by a small extension of the ITER superconducting magnet technology. Regarding the core plasma performance, the same magnetic field configuration and beta profile as those in the past design study FFHR-d1B [9], were assumed. Plasma performance (fusion gain $Q$ ) and the total stored magnetic energy of the superconducting system,

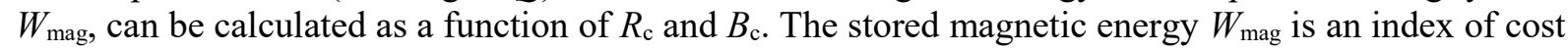

(a)

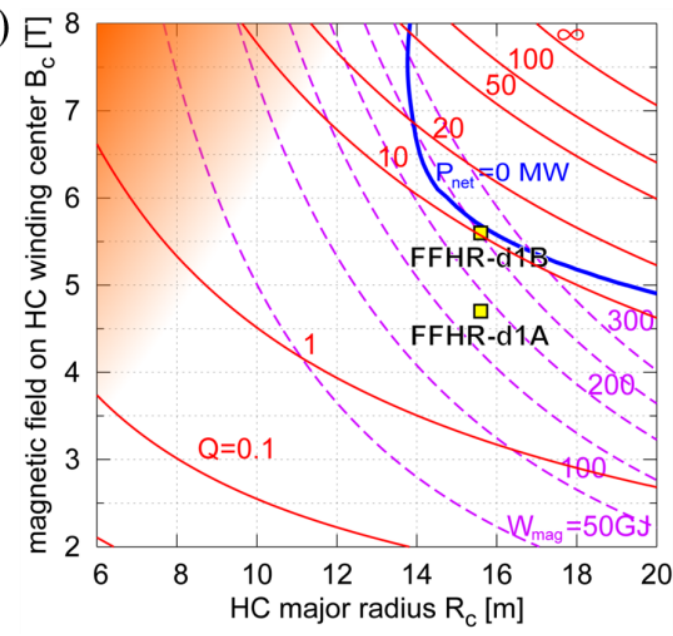

(b)

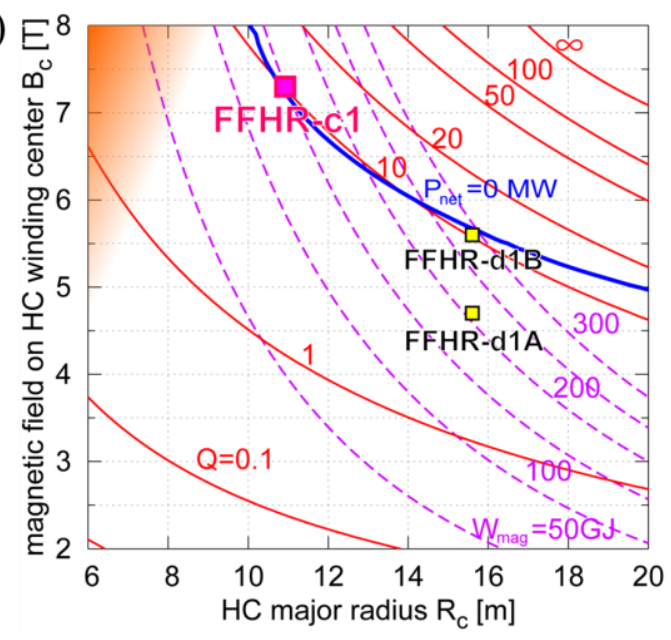

Figure 1. Result of the design window analysis with the following assumptions: (a) coil current density of $25 \mathrm{~A} / \mathrm{mm}^{2}$ without NITA coils, (b) coil current density of $48 \mathrm{~A} / \mathrm{mm}^{2}$ with NITA coils. The contour of the stored magnetic energy (purple broken lines) and fusion gain (red thin solid lines) are plotted. The thick blue line corresponds to the electric break-even condition. Shaded region corresponds to the region with too high nuclear heating on superconducting coils. 
and technological difficulty of the superconducting magnet system. As shown in Fig. 1(a), $W_{\text {mag }}$ can be reduced by maintaining the same fusion gain by decreasing $R_{\mathrm{c}}$ and increasing $B_{\mathrm{c}}$ at the same time. The cost of the most of other plant systems depends on its mass or volume. Therefore, the construction cost and the construction period of the reactor can be reduced if $R_{\mathrm{c}}$ is decreased by maintaining the fusion gain. However, both the decrease of $R_{\mathrm{c}}$ and the increase of $B_{\mathrm{c}}$ lead to the decrease in the thickness of the blanket module if $j_{\mathrm{c}}$ is fixed. The decrease in the thickness of the blanket module causes the increase in the neutron flux to the superconducting magnet system, resulting in high nuclear heat generation. The shaded region in Fig. 1(a) corresponds to the design region with too high nuclear heat $(10 \mathrm{~mW} / \mathrm{cc})$, which cannot be handled by any combination of superconducting material and coolant. Even if the nuclear heat is smaller than $10 \mathrm{~mW} / \mathrm{cc}$, the increase in the nuclear heat leads to the increase of the power consumption of the cryogenic system, resulting in the limitation of the design window from the viewpoint of plant power balance. That is because the contour of $P_{\mathrm{e}, \text { net }}=0$ deviates from the contours of fusion gain in the region of $R_{\mathrm{c}}<14 \mathrm{~m}$. Because the power consumption of other plant systems is also a function of $R_{\mathrm{c}}$ and $B_{\mathrm{c}}$, the design point of FFHR-d1B becomes a 'minimum' $R_{\mathrm{c}}$ that can achieve the condition of $P_{\mathrm{e}, \text { net }}>0$ as a result. However, new design concepts introduced as a challenging option of FFHR-d1A, the adoption of high temperature superconductor and the NITA coil, expand the reachable design window to the region with a smaller $R_{\mathrm{c}}$. Finally, it was found that the originally proposed design point $\left(R_{\mathrm{c}}=10.92 \mathrm{~m}, B_{\mathrm{c}}=7.3 \mathrm{~T}\right)$ will be a minimum design point that can achieve $P_{\mathrm{e}, \text { net }}>0$ with $Q=10$ if the adoption of NITA coils and the condition of $j_{\mathrm{c}}=48 \mathrm{~A} / \mathrm{mm}^{2}$ are assumed, as shown in Fig. 1(b). This design point has a fusion power of $P_{\text {fus }} \sim 400 \mathrm{MW}$. The comparison of the primary design parameters of FFHR-c1 and FFHR-d1 is summarised in Table 1.

Table 1. Comparison of primary design parameters of FFHR-c1 and FFHR-d1

\begin{tabular}{ccc}
\hline & FFHR-c1 & FFHR-d1A/d1B \\
\hline Major radius $R_{\mathrm{c}}[\mathrm{m}]$ & 10.92 & 15.6 \\
Magnetic field strength $B_{\mathrm{c}}[\mathrm{T}]$ & 7.3 & $4.7 / 5.6$ \\
Fusion power $P_{\text {fus }}[\mathrm{MW}]$ & $\sim 400$ & $\sim 3000^{*}$ \\
Peak beta value $\beta_{0}[\%]$ & $\sim 3.0$ & $\sim 8.0^{*} / 5.6^{*}$ \\
Net electric output $P_{\mathrm{e}, \text { net }}[\mathrm{MW}]$ & $>0$ & $\sim 1000^{*}$ \\
Fusion gain & $\sim 13$ & $\infty^{*}$ \\
Ratio of alpha heating power to & $\sim 2.5$ & $\infty^{*}$ \\
$\quad$ auxiliary heating power & 48 & 25 \\
Current density $j_{\mathrm{c}}\left[\mathrm{A} / \mathrm{mm}^{2}\right]$ & $\sim 150$ & $\sim 180 / \sim 250$ \\
Stored magnetic energy $W_{\text {mag }}[\mathrm{GJ}]$ & $\sim 10$ & $>30$ \\
Plant lifetime $[$ years $]$ &
\end{tabular}

* With an improvement in the plasma performance

\section{Core plasma physics analysis}

\subsection{Estimation of achievable fusion gain}

The design window analysis of the previous section is based on the core plasma performance estimated from the previous design FFHR-d1. Therefore, achievable fusion gain should be examined with the actual design parameter of FFHR-c1 to confirm the feasibility of the core plasma design. For this purpose, integrated physics analysis has been conducted. In this analysis, the same magnetic configuration as that of the previous study for FFHR-d1B [9] (with a high aspect ratio $A_{p} \sim 7$ and inwardshifted magnetic axis position in vacuum equilibrium with the ratio of the magnetic axis position $\underline{R}_{\mathrm{ax}, \mathrm{vac}}$ 
to $R_{\mathrm{c}}$ is $3.5 / 3.9$ ) was assumed. In this analysis, temperature and density profiles of electrons and ions were determined by the model based on the LHD experimental observation that is given in Ref. [10]. First, the pressure profiles of electrons and ions in the reactor are given as a function of normalised minor radius $\rho$ by

$$
\begin{aligned}
& p_{e}(\rho)=\gamma_{\mathrm{DPE} *, e} \hat{p}(\rho) P_{\mathrm{abs}, e}^{0.4} B^{0.8} n_{e}(\rho)^{0.6}, \\
& p_{i}(\rho)=\gamma_{\mathrm{DPE} *, i} \hat{p}(\rho) P_{\mathrm{abs}, i}^{0.4} B^{0.8} n_{i}(\rho)^{0.6} .
\end{aligned}
$$

where $B, n_{e}$ and $n_{i}$ are the magnetic field strength, the electron density and the ion density, respectively. In FFHR-c1, only electron cyclotron heating $(\mathrm{ECH})$ is assumed to be used as an external heating source by considering its small effect on the blanket coverage, efficiency of the core heating and capability of the protection of the device from the direct irradiation of fusion neutrons. Then the terms of the absorbed power in Eqs. (1) and (2) are given respectively as

$$
\begin{aligned}
& P_{\mathrm{abs}, e}=\eta_{\alpha} P_{\alpha}+\eta_{\mathrm{aux}, e} P_{\mathrm{aux}, e}-P_{\mathrm{rad}}-P_{e i}, \\
& P_{\mathrm{abs}, i}=P_{e i} .
\end{aligned}
$$

In Eqs. (3) and (4), $\eta_{\alpha}$ and $\eta_{\text {aux,e }}$ are absorption efficiency of the alpha heating power and auxiliary heating power to electrons, respectively. The power gain/loss term $q \Gamma E_{r}$ which arises from a particle species with charge $q$ and particle flux density $\Gamma$ in the presence of radial electric field $E_{r}$ is ignored because its magnitude (estimated to be $\sim 1 \mathrm{~kW} / \mathrm{m}^{3}$ at maximum) is much smaller than those of other terms $\left(>20 \mathrm{~kW} / \mathrm{m}^{3}\right)$. The terms of the heating power and the power loss $P_{X}(X=\alpha$, aux, $\operatorname{rad}, e i)$ are calculated from the radial profiles $Q_{X}(\rho)$ :

$$
P_{X}=\int_{0}^{1} Q_{X}(\rho) \frac{\mathrm{d} V}{\mathrm{~d} \rho} \mathrm{d} \rho .
$$

The equipartition power from electrons to ions is calculated by

$$
Q_{e i}(\rho)=\frac{1.5 k_{B}\left\{T_{e}(\rho)-T_{i}(\rho)\right\} n_{e}(\rho)}{\tau_{e i}^{\varepsilon}(\rho)},
$$

where $k_{B}$ and $\tau_{e i}^{\varepsilon}$ are Boltzmann constant and electron-ion energy relaxation time, respectively. In Eqs. (1) and (2), $\hat{p}(\rho)$ is the gyro-Bohm normalised pressure profile defined as

$$
\hat{p}(\rho)=\frac{p_{e, \exp }(\rho)}{P_{\mathrm{abs}, \exp }^{0.4} B_{\exp }^{0.8} n_{e, \exp }(\rho)^{0.6}},
$$

where the subscript 'exp' denotes that the parameters are obtained from the reference LHD experimental data. $\gamma_{\mathrm{DPE} *, e}$ and $\gamma_{\mathrm{DPE} * i}$ in Eqs. (1) and (2) are the confinement improvement factor related to the peakedness of the heating profile [11] for electrons and ions, respectively. The definition is given by

$$
\begin{aligned}
& \gamma_{\mathrm{DPE} *, x}=\left\{\frac{\left(P_{\mathrm{dep}} / P_{\mathrm{dep} 1}\right)_{\mathrm{avg}, x}}{\left(P_{\mathrm{dep}} / P_{\mathrm{dep} 1}\right)_{\mathrm{avg}, \mathrm{exp}}}\right\}^{0.6}, \\
& \left(P_{\mathrm{dep}} / P_{\mathrm{dep} 1}\right)_{\mathrm{avg}, x}=\int_{0}^{1} \frac{P_{\mathrm{dep}, x}(\rho)}{P_{\mathrm{dep}, x}(1)} \mathrm{d} \rho, \\
& P_{\mathrm{dep}, x}(\rho)=\int_{0}^{\rho} P_{\mathrm{abs}, x}\left(\rho^{\prime}\right)\left(\frac{\mathrm{d} V}{\mathrm{~d} \rho^{\prime}}\right) \mathrm{d} \rho^{\prime},
\end{aligned}
$$


for $x=e, i . P_{\mathrm{dep}, x}(\rho)$ is the deposition profile of the absorbed power that given by Eqs. (3) and (4). In this study, density profile is determined by the calculation with the model based on the LHD experimental observation because there is no definitive way to give the density profile in a reactor condition. The density profile was obtained by solving a diffusion equation with the following three assumptions: spatially-constant diffusion coefficient which is proportional to absorbed power density, no advection flow, and particle source profile which is the same as the ablation profile of the pellet calculated by the neural gas shielding (NGS) model. Time evolution of typical LHD pellet discharges can be reproduced by the calculation with these assumptions [12]. Although NBI heating was used for most of LHD discharges, it was found that the density profile is determined only by transport and NBI has not effect on density peaking [13]. Consequently, flat profile with a shoulder structure around $\rho=$ 0.7 (as shown in Fig. 2 of Ref. [10]) was obtained and used in this study. Regarding the ion density profile, helium ash fraction of 5\% is assumed and absolute value of the density of deuterons and tritons are given to be 0.45 times that of electrons at any radial position. No other impurity was considered in this study.

The reference pressure profile is shown in Fig. 2. This profile was obtained from LHD experimental data with the peak beta value close to its MHD stability limit (\#115772@t=3.533 s). The peak normalised pressure is higher than that in the previous study even though the flattening of the pressure profile is observed around the normalised minor radius of $\rho=0.4$. Here two fittings were conducted. One is the fitting using all data (solid line in Fig. 2) and the other is the fitting using the data with $\rho>0.7$ (dotted line in Fig. 2), which predicts the peak beta value if the pressure flattening did not take place. The results are given in Fig. 3. The reachable operation region of the LHD experiment is limited by two constraints: MHD stability limit and energy confinement property. The MHD stability limit can be well described by Mercier index $D_{I}$ at $m / n=1 / 1$ rational surface. In high beta discharges in the LHD experiment, it has been observed that a low- $n$ MHD mode that causes core pressure collapse emerges when

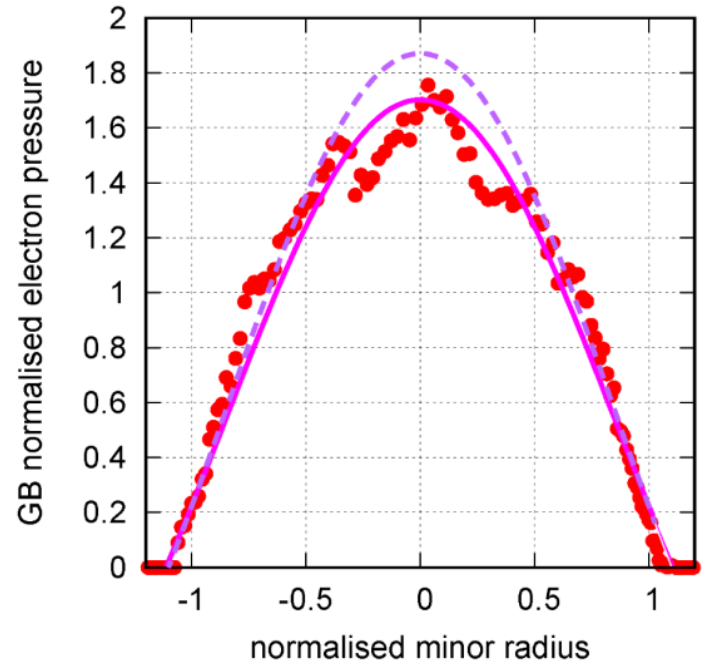

Figure 2. Radial profile of gyro-Bohm normalised pressure of the reference experimental data (circles). The lines are fitting curve used in this analysis using all data (solid line) and the data with the normalised minor radius of $\rho>0.7$ (broken line). $D_{I}$ exceeds $0.2-0.25$, and this condition corresponds to the theoretical prediction $[14,15]$. On the other hand, the growth rate of the instability decreases with increasing magnetic Reynolds number [16], which becomes larger in the reactor condition. Some experimental results indicate that the MHD stability is maintained with further larger value of the Mercier index. Therefore, $D_{I}<0.3$ was selected as the constraints of the MHD stability in this analysis. Regarding the energy confinement property, the thermal diffusivity evaluated from the LHD experimental results qualitatively agrees with the prediction by neoclassical theory but the absolute value is $2-3$ time higher due to the existence of anomalous transport [17]. The constraint on the energy confinement was quantified by the ratio of the neoclassical energy loss to the total absorbed power, $Q_{\text {neo }} S / P_{\text {abs. }}$. The power balance should be satisfied on every flux surface. Therefore, the maximum value of $Q_{\text {neo }} S / P_{\text {abs }}$ over the minor radius, $\left(Q_{\text {neo }} S / P_{\text {abs }}\right)_{\max }$, was used as an index of the energy confinement and $\left(Q_{\text {neo }} S / P_{\text {abs }}\right)_{\max }<0.5$ was assumed in this analysis (corresponds to the condition that the energy loss by anomalous transport is the same as the energy loss by the neoclassical transport). Calculations were performed by the detailed physics analysis tools that have been developed for the integrated transport analysis suite TASK3D-a [18]. MHD equilibrium and Mercier index $D_{I}$ are calculated by $3 \mathrm{D}$ equilibrium calculation code VMEC [19]. The neoclassical energy flux is calculated using GSRAKE code [20]. In the calculation of GSRAKE, pure deuterium plasma $(A=2)$ was assumed because the code can only 
handle a single ion species with an integer mass number. The ambipolar radial electric field was selfconsistently solved in the calculation so that the equality of the particle flux of ions and electrons was satisfied on every flux surface.

Figure 3 shows the dependence of $D_{I},\left(Q_{\text {neo }} S / P_{\text {abs }}\right)_{\max }$, beta value at the magnetic axis $\beta_{0}$ and fusion gain $Q$ on the electron density and temperature. The achievable maximum fusion gain is $\sim 15$ ( $P_{\text {fus }} \sim 370 \mathrm{MW}$ with an ECH power of $P_{\mathrm{ECH}} \sim 25 \mathrm{MW}$ ) with the original peak normalised pressure, whereas the fusion gain remains $\sim 10$ with the higher peak normalised pressure (see the star symbols in Fig. 3(a) and (b)). This is because higher normalised pressure leads to less external heating power amount and the reduction of the total absorbed power especially around the core region, whereas the profile of the neoclassical energy loss is almost uniquely determined by the profiles of electron density and temperature. Therefore, higher normalised pressure does not necessarily lead to higher fusion gain. The achievable fusion gain increases if the anomalous transport is suppressed. In the case of the original normalised pressure profile, the fusion gain can slightly increase to $\sim 18$. The fusion gain can increase to $\sim 30$ in the case of the higher normalised pressure.

(a)

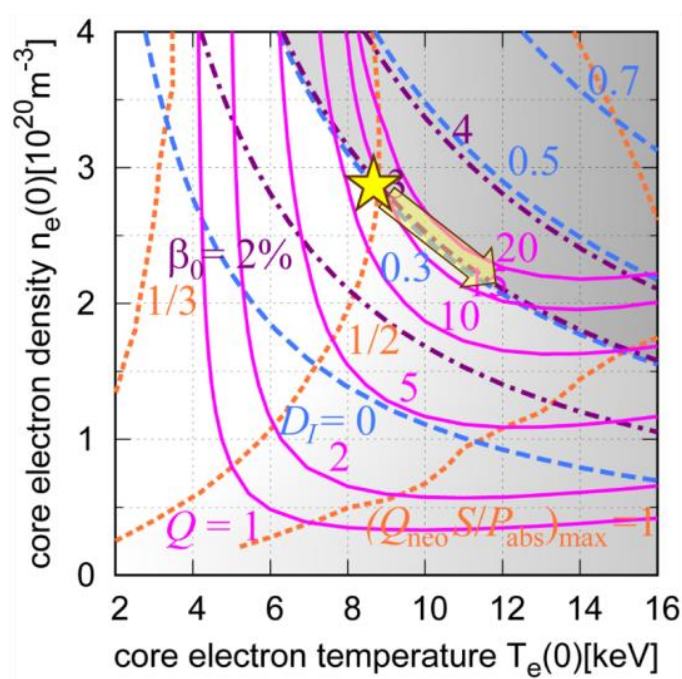

(b)

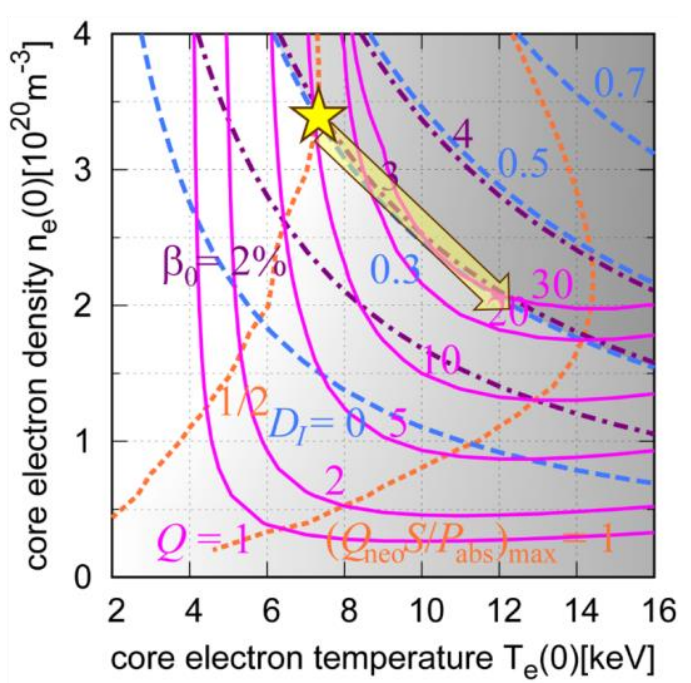

Figure 3. Operation region of FFHR-c1 with the pressure profile of (a) solid curve in Fig.2 and (b) dotted curve in Fig. 2. The contours of the fusion gain (magenta solid curve), the peak beta value (maroon dashdotted curve), the Mercier index (blue broken curve) and the ratio of the neoclassical energy loss to the total absorbed power (orange dotted curve) are plotted. The region without the shading corresponds to the operation regime with the physics conditions that have already been confirmed by the LHD experiment. Star symbol corresponds to the operation point with the maximum achievable fusion gain under the physics condition that has already confirmed in the LHD experiment. Arrow shows the extension of the operation regime when anomalous transport is suppressed.

Consequently, fusion gain of $Q \sim 15$ can be achieved with the physics conditions that has already confirmed by the LHD experiment. Further larger fusion gain can be expected if the anomalous transport is suppressed. However, the beta value achieved in the LHD experiment depends on the magnetic axis position [21]. In this analysis, $\beta_{0} \sim 3 \%$ at the operation point of $Q>15$, whereas and experimentally achieved value is $\beta_{0} \sim 2.5 \%$ with the magnetic axis position of this operation point calculated by VMEC. Therefore, another approach including the optimisation of the magnetic configuration is desired to ensure the achievement of the sufficient fusion gain for net electricity generation. 


\subsection{Examination of modification of the helical coil winding law}

Regarding the configuration optimisation, several sophisticated theoretical works have been conducted using optimisation codes [22-24]. However, there is a possibility of simultaneous improvement in the MHD stability and neoclassical transport by a minor change in the magnetic configuration, i.e., modification of the winding law of the helical coil.

Trajectory of the helical coils of the LHD-type helical reactor is described by the following formulae.

$$
\begin{aligned}
& R=R_{\mathrm{c}}+a_{\mathrm{c}} \cos \theta, \\
& Z=Z_{\mathrm{c}} \sin \theta .
\end{aligned}
$$

The relation between the poloidal angle $\theta$ and the toroidal angle $\phi$ is defined by the following formula:

$$
\theta=\frac{m}{\ell} \phi+\alpha \sin \left(\frac{m}{\ell} \phi\right),
$$

where $m, \ell$ and $\alpha$ are toroidal pitch number, the number of helical coil and helical pitch modulation parameter, repectively. The trajectory and cross-sectional shape of the helical coils with different $\alpha$ are plotted in Fig. 4.

(a)

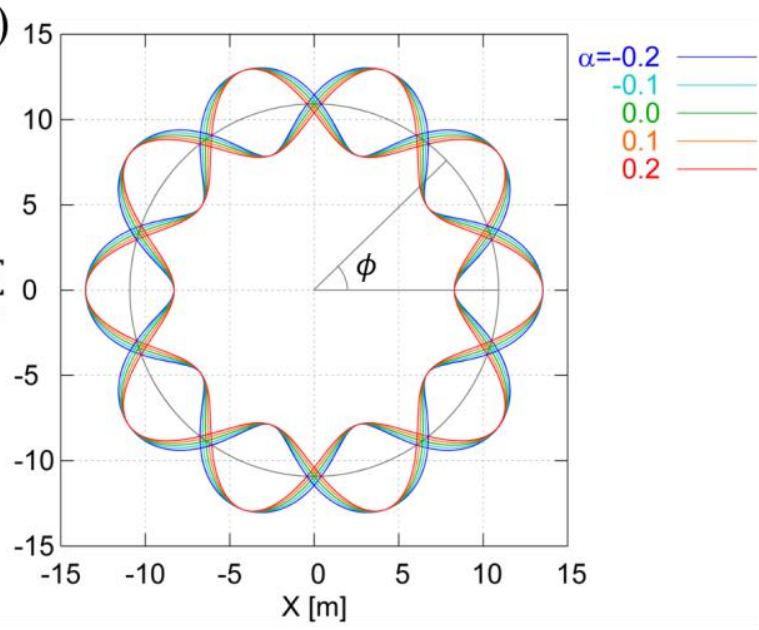

(b)

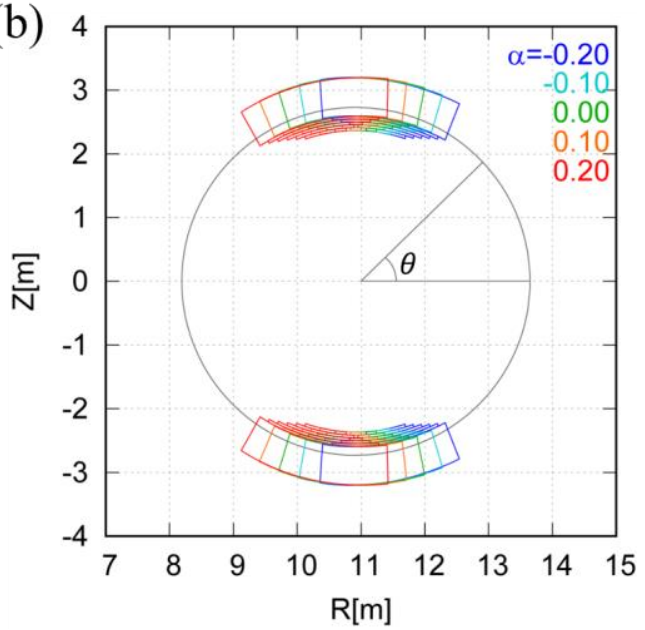

Figure 4. (a) Top view of trajectory of helical coils and (b) cross-sectional view of the helical coils at the toroidal cross-section at which the plasma has horizontally-elongated cross-sectional shape $\left(\phi=18^{\circ}\right)$ with different helical pitch modulation parameters $\alpha$. The trajectory of the helical coils shifts toward the outboard side of the torus with the decrease of $\alpha$.

In this study, dependence of the physics constraints on the helical pitch modulation parameter $\alpha$ has been examined. Figure 5 shows the comparison of the shape of the magnetic flux surface at the poloidal cross-section in which the plasma has a vertically elongated cross-sectional shape. In MHD equilibrium analysis with a finite pressure, the same pressure profile as that in the analysis of the previous section was assumed. In this analysis, the current of the two pairs of poloidal (vertical field) coils was set so that the magnetic axis position and the ratio of quadrupole field component of the vertical field coils to that of the helical coils are $R_{\mathrm{ax}, \mathrm{vad}} / R_{\mathrm{c}}=3.6 / 3.9$ and $B_{q \text {, poloidal }} / B_{q}$, helical $=0.72$, respectively. The value of $B_{q}$, poloidal $/ B_{q}$,helical $=0.72$ is the same as that in the previous design, FFHR-d1B. The scan range of $\alpha$ was set from -0.2 to 0.2 because an appropriate comparison becomes difficult when $\alpha$ is outside this range due to the strong deformation of the plasma shape and the significant decrease of the plasma volume. As shown in Fig. 5, cross-sectional shape transforms to ' $\mathrm{D}$ '-shape if $\alpha$ increases. If $\alpha$ becomes negative, 
the cross-sectional shape changes to become 'inverse-D' shape. The plasma volume has a maximum at $\alpha=0.0$.
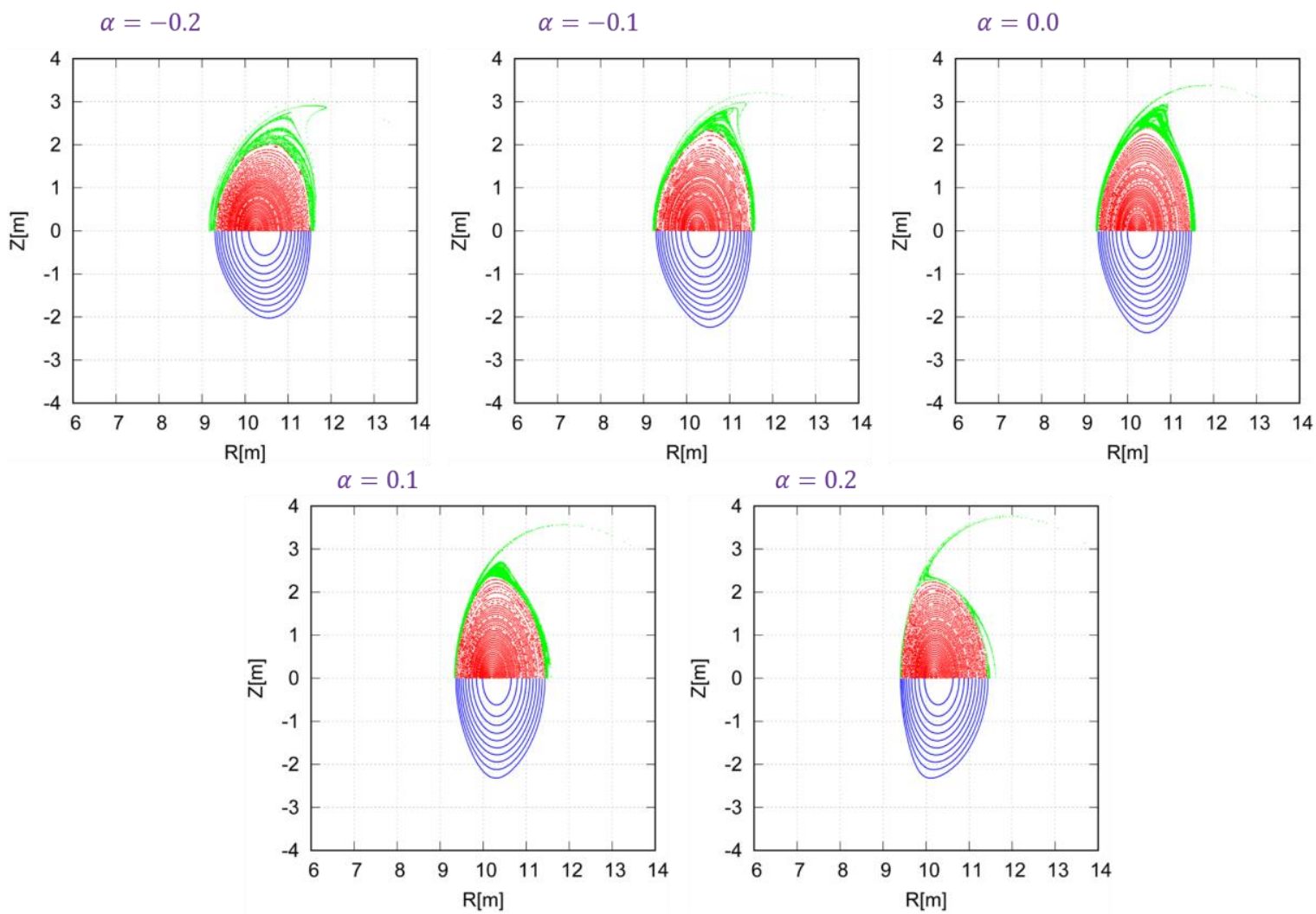

Figure 5. Magnetic surface shape of vacuum equilibrium calculated by field line tracing code (upper) and finite-beta equilibrium obtained by VMEC with the same fixed boundary shape as that of the last closed flux surface of the vacuum equilibrium (lower) for $\alpha=-0.2$ (top left) to 0.2 (bottom right). Magnetic axis positions of vacuum equilibrium are set to $R_{\mathrm{ax}, \mathrm{vac}} / R_{\mathrm{c}}=3.6 / 3.9$.

It is known that HINT2 code [25], which is the 3D equilibrium code of an initial value solver based on the relaxation method without the assumption of nested flux surfaces, can reproduce the experimental results better than VMEC. In this analysis, however, finite-beta equilibria were calculated by VMEC code because of its low computational cost. All equilibria in this analysis were obtained by assuming the same LCFS shape as that in the vacuum equilibrium. In the previous design FFHR-d1, it has shown that a high-beta equilibrium with a similar LCFS shape as that in the vacuum equilibrium can be achieved by optimising the magnetic axis position. Of course the LCFS shape in a finite-beta equilibrium is not exactly same as that in the vacuum equilibrium. The VMEC equilibria strongly depends on the assumed LCFS shape. Therefore, absolute values in this analysis should be examined by the detailed calculation including HINT2 calculation. However, this analysis can be a semiquantitative evaluation of the dependence on $\alpha$.

Figure 6 shows the radial profiles of rotational transform $t / 2 \pi$, Mercier index $D_{I}$ and the maximum value over the minor radius of the ratio of neoclassical energy loss to the total absorbed power $Q_{\text {neo }} S / P_{\text {abs }}$ for different $\alpha$. The core rotational transform decreases with the decrease of $\alpha$. Regarding $D_{I}$ at $m / n=1 / 1$ rational surface, it slightly decreases the decrease in $\alpha$. Regarding the neoclassical power loss, it has the minimum value around $\alpha=0.0$. Figure 7 shows $\alpha$ dependence of $D_{I},\left(Q_{\text {neo }} S / P_{\text {abs }}\right)_{\max }$, plasma volume $V_{p}$ and the minimum distance between the helical coil and the plasma $\Delta_{\mathrm{c}-\mathrm{p}}$ (including the stochastic field region). It was found that both $D_{I}$ at $m / n=1 / 1$ rational surface and $\left(Q_{\text {neo }} S / P_{\text {abs }}\right)_{\max }$ decrease by changing $\alpha$ from 0.1 to $0.0 . D_{I}$ and $\left(Q_{\text {neo }} S / P_{\text {abs }}\right)_{\max }$ are also a function of the magnetic axis position. The calculation result with slightly inward-shifted magnetic axis position, $R_{\mathrm{ax}, \mathrm{vad}} / R_{\mathrm{c}}=3.55 / 3.9$, are also plotted in Fig. 7 . In LHD experiment, inward shift of the magnetic axis position improves the energy confinement but 
(a)

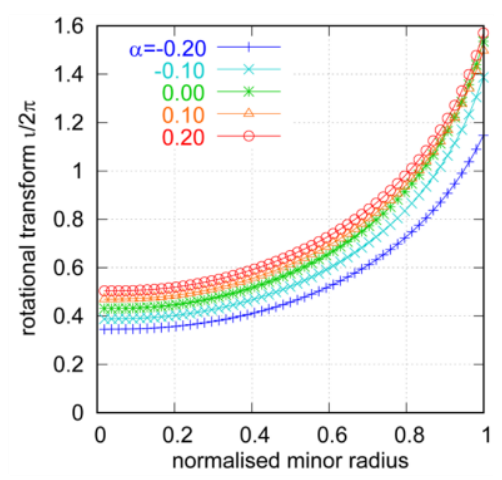

(b)

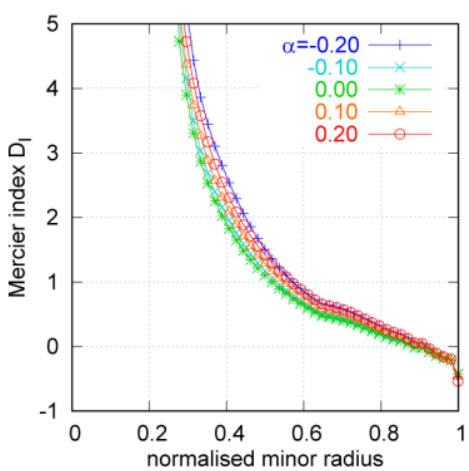

(c)

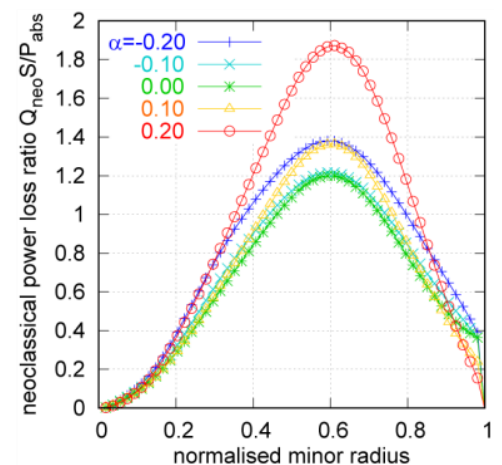

Figure 6. Radial profile of (a) rotational transform, (b) Mercier index and (c) ratio of neoclassical power loss to the total absorbed power with different helical pitch modulation parameters $\alpha$ with inward shifted magnetic axis position $\left(R_{\mathrm{ax}, \mathrm{vad}} / R_{\mathrm{c}}=3.6 / 3.9\right)$.

deteriorates the MHD stability. This tendency is common to different $\alpha$ values. As a result, significant improvement of the neoclassical power loss is expected without the increase of $D_{I}$ by simultaneous change of $\alpha$ and the magnetic axis position (decrease of $\alpha$ from 0.1 to 0.0 and inward shift of the magnetic axis from $R_{\mathrm{ax}, \mathrm{vac}} / R_{\mathrm{c}}=3.6 / 3.9$ to 3.55/3.9). Of course the decrease of $\alpha$ is not the answer to everything. In the case of smaller $\alpha$, rotational transform in the core region becomes low and $t / 2 \pi=0.5$ rational surface emerges. Thus, MHD instability in the core region may become problematic. As shown in Fig. 7, the distance between the helical coils and the plasma $\Delta_{\mathrm{c}-\mathrm{p}}$ decreases with the decrease of $\alpha$ and the inward shift of the magnetic axis. It leads to the decrease of the space for the blanket modules. To maintain the same neutron shielding performance as that in the case of $\alpha=0.1$, design optimisation of the blanket modules or coils is required. However, it can be concluded that simultaneous improvement in the MHD stability and neoclassical transport can be expected by a modification of the winding law, especially the helical pitch modulation parameter $\alpha$.

\section{Summary}

Design study of the LHD-type helical fusion reactor which enables a year-long steady-state operation with self-sufficiency of electricity and tritium fuel has been conducted. The proposed design point with $R_{\mathrm{c}}=10.92 \mathrm{~m}$ and $B_{\mathrm{c}}=7.3 \mathrm{~T}$ has been confirmed by the design window analysis using the systems code HELIOSCOPE. It was found that this design point can achieve fusion gain of $\sim 15$ with a fusion power

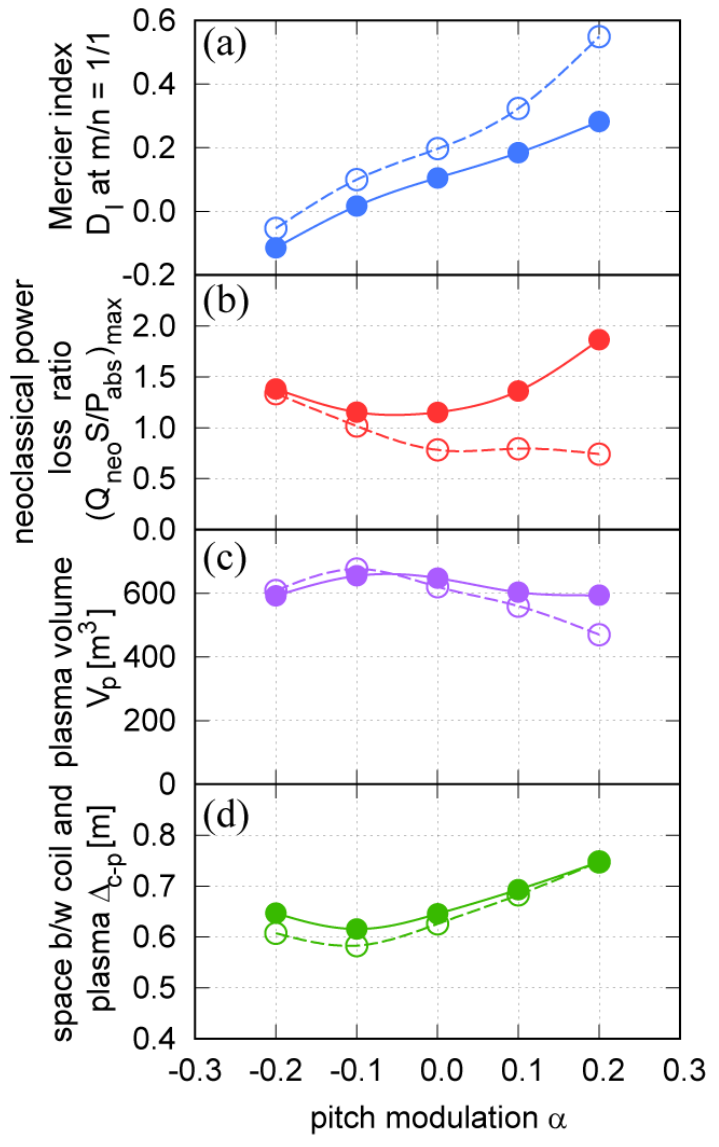

Figure 7. Dependence of (a) Mercier index, (b) ratio of neoclassical power loss to the total absorbed power, (c) plasma volume and (d) minimum distance between helical coil and plasma on the helical pitch modulation parameter $\alpha$. Solid line/closed symbols and broken line/open symbols correspond to the magnetic axis position of $R_{\mathrm{ax}, \mathrm{vac}} / R_{\mathrm{c}}=3.6 / 3.9$ and $R_{\mathrm{ax}, \mathrm{vac}} / R_{\mathrm{c}}=3.55 / 3.9$, respectively. 
of $\sim 370$ MW by the integrated physics analysis of the core plasma. Further larger fusion gain can be expected if anomalous transport is suppressed.

There still remain several engineering and physics issues. Especially, design of superconducting coils with a high current density of $48 \mathrm{~A} / \mathrm{mm}^{2}$ is a challenging issue. In this regard, innovative concepts including an insulation-less conductor concept have been being intensively studied. Examination of the equipment layout, construction scenario and maintenance scenario, as well as the detailed design analysis of each component (e.g., structural analysis of the coil supporting structure, thermohydraulic and neutronics analysis of the blanket) have been also conducted to ensure the consistent system design Achievement of further higher peak beta value of $\sim 3 \%$ with an inward-shifted configuration is an important physics issue. Regarding this issue, a combination of 'minor' modification of the winding law of helical coils, especially a slight decrease in the helical pitch modulation parameter $\alpha$ and the optimisation of the magnetic axis position can be a solution. Consequently, the design feasibility of a compact LHD-type helical reactor that can satisfy the requirements on Japanese fusion DEMO, steadystate electricity generation above several hundred MW, tritium fuel self-sufficiency and practical availability, has been shown.

\section{Acknowledgements}

This work is supported by the budget NIFS10UFFF011 of National Institute for Fusion Science and MEXT/JSPS KAKENHI Grant Number 24760704. The authors also would appreciate the members of the Fusion Engineering Research Project and the Numerical Simulation Reactor Research Project in NIFS for giving valuable comments and advices.

\section{References}

[1] Komori A. et al 2010 Fusion Sci. Technol. 581

[2] Sagara A. et al 2012 Fusion Eng. Des. 87594

[3] Yanagi N. et al 2014 IEEE Trans Appl. Supercond. 244020805

[4] Yanagi N. et al 2016 Plasma Fusion Res. 112405034

[5] Miyazawa J. et al 2017 Fusion Eng. Des. 125227

[6] Miyazawa J. et al 2017 Plasma Fusion Res. 121405017

[7] Miyazawa J. et al 2018 Fusion Eng. Des. 1361278

[8] Goto T. et al 2011 Nucl. Fusion 51083045

[9] Goto T. et al 2017 Nucl. Fusion 57066011

[10] Goto T. et al 2018 Plasma Phys. Control. Fusion 60074001

[11] Miyazawa J. et al 2014 Nucl. Fusion 54013014

[12] Sakamoto R. et al 2015 IEEE Trans. Plasma Sci. 442915.

[13] Tanaka K. et al 2008 Plasma Fusion Res. 3 S1069.

[14] Yamada H. et al 2004 J. Plasma Fusion Res. Ser. 651

[15] Sakakibara S. et al 2006 in Fusion Energy 2006 (Proc. 21st Int. Conf. Chengdu, China, 2006) (Vienna: IAEA) CD-ROM file [EX/7-5] and http://wwwnaweb.iaea.org/napc/physics/FEC/FEC2006/html/index.htm

[16] Sakakibara S. et al 2008 Plasma Phys. Control. Fusion 50124014

[17] Yamada H. et al 2010 Phys. Rev. Lett. 841216

[18] Yokoyama M. et al 2017 Nucl. Fusion 57126016

[19] Hirshman S.P. et al 1983 Phys. Fluids 263553

[20] Beidler C.D. et al 1995 Plasma Phys. Control. Fusion 37463

[21] Komori A. et al 2009 Nucl. Fusion 49104015

[22] Brown T. et al 2015 IEEE 26th Symposium on Fusion Engineering (SOFE) 1-6

[23] Landreman M. et al 2017 Nucl. Fusion 57046003

[24] Zhu C. et al 2018 Plasma Phys. Control. Fusion 60065008

[25] Suzuki Y. et al 2006 Nucl. Fusion 46 L19 